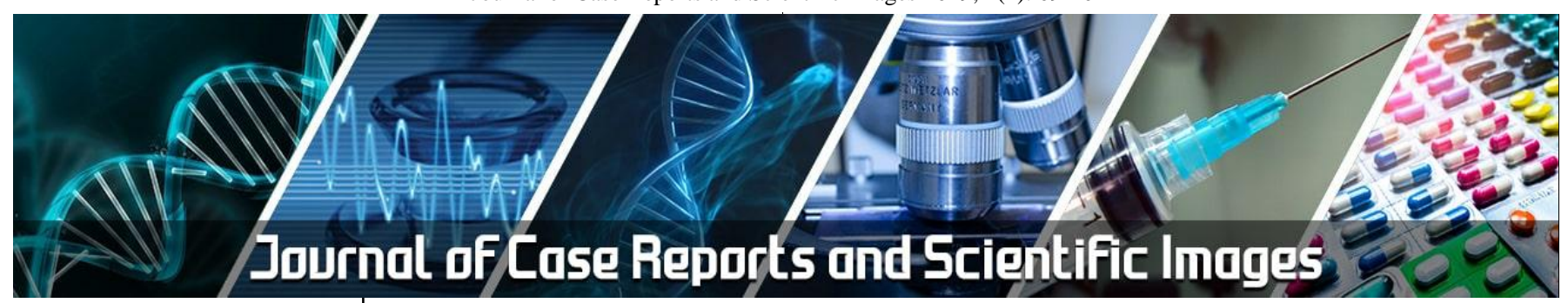

E-ISSN: 2708-0064 P-ISSN: 2708-0056 www.allcasereports.com JCRSI 2019; 1(2): 09-10 Received: 15-05-2019 Accepted: 18-06-2019

Reza Bastian

Department of Scientific, Research Center for Physics, Indonesian Institute of Sciences, Indonesia
Corresponding Author: Reza Bastian

Department of Scientific, Research Center for Physics, Indonesian Institute of Sciences, Indonesia

\section{Familial primary open-angle glaucoma: A case report}

\section{Reza Bastian}

DOI: $\underline{\text { https://doi.org/10.33545/27080056.2019.v1.i2a.10 }}$

\begin{abstract}
Glaucoma affects more than 70 million people worldwide with approximately $10 \%$ being bilaterally blind, making it the leading cause of irreversible blindness in the world. Primary open-angle glaucoma (POAG) is a disease that is often inherited. We reported a case of Primary open-angle glaucoma in 27 years old male patient.
\end{abstract}

Keywords: Blindness, inherited, primary open-angle glaucoma

\section{Introduction}

Glaucoma is a devastating, worldwide disease. In just the United States there are over 2 million estimated cases with 120,000 of those responsible for blindness ${ }^{[1]}$. Unfortunately, only half of those 2 million people are estimated to be aware of their condition ${ }^{[2]}$. Angleclosure glaucoma is an acute condition associated with rapid onset of severe pain, erythema and visual loss, and thus emergent surgery is employed as a therapeutic intervention ${ }^{[3]}$. In contrast, open angle glaucoma, the most common subtype of glaucoma, is an insidious process that results in slow, progressive, and irreversible loss of the visual field. Early stages of open angle glaucoma may be asymptomatic, allowing the disease to go unnoticed and progress to optic nerve damage ${ }^{[4]}$.

Glaucoma affects more than 70 million people worldwide with approximately $10 \%$ being bilaterally blind, making it the leading cause of irreversible blindness in the world. Glaucoma can remain asymptomatic until it is severe, resulting in a high likelihood that the number of affected individuals is much higher than the number known to have it ${ }^{[5]}$.

Primary open-angle glaucoma (POAG) is a disease that is often inherited, but the rules governing its inheritance are inconclusive. Genetic analysis technology was heralded as a solution to the POAG genetic conundrum. Scientists have identified MYOC, OPTN, WDR36, and CYP1B1 as being related to POAG, and found that mutations in these genes play a key role in POAG onset. Although familial patients are the best research subjects for inherited diseases, genes involved in POAG pathogenesis show regional and ethnic differences ${ }^{[6]}$. We reported a case of Primary open-angle glaucoma in 27 years old male patient.

\section{Case Report}

A 27 years old male patient referred to Eye department with reduction in progressive visual acuity in the right eye since 2 years. Patient was medically fit.

Ophthalmological examination revealed right eye visual acuity of 20/70. There were no apparent pathological changes in the anterior segment of either eye. Gonioscopy examination showed normal appearance and open anterior chamber angle without peripheral anterior synechiae. Fundus examination showed an acquired optic nerve pit in both eyes. Cup/disk (C/D) asymmetry was 0.5 and 0.7 in the right and left eyes, respectively. Intraocular pressure (IOP) in the right eye was $37.4 \mathrm{mmHg}$. Visual field in the right eye showed nasal step. On the basis of findings, we gave the diagnosis of POAG in right eye. Patient underwent trabeculectomy. On taking family history, we found that 8 people in this family had same complaint. The age at diagnosis was between 15 and 42 years. Collectively, their visual field impairments were mostly early or metaphase-related, and the IOP ranged from 23.21 to $39.40 \mathrm{mmHg}$. Their prognoses were satisfactory after undergoing an operation or receiving medication. 


\section{Discussion}

Glaucomas can be classified into 2 broad categories: openangle glaucoma and angle-closure Glaucoma ${ }^{[7]}$. In the United States, more than $80 \%$ of cases are open-angle glaucoma; however, angle-closure glaucoma is responsible for a disproportionate number of patients with severe vision loss. Both open-angle and angle-closure glaucoma can be primary diseases. Secondary glaucoma can result from trauma, certain medications such as corticosteroids, inflammation, tumor, or conditions such as pigment dispersion or pseudo-exfoliation ${ }^{[8]}$.

The risk of glaucoma was highest when examination revealed an increased cup-disk ratio (CDR), CDR asymmetry, disc hemorrhage, or elevated intraocular pressure. Primary open-angle glaucoma was also more likely when there was a family history of the disease, black race, or advanced age. The primary care physician also should be aware of the risk of developing glaucoma in patients being treated with systemic or topical corticosteroids ${ }^{[9]}$. We reported a case of Primary open-angle glaucoma in 27 years old male patient.

Although the pathogenesis of glaucoma is not fully understood, the level of intraocular pressure is related to retinal ganglion cell death. The balance between secretion of aqueous humor by the ciliary body and its drainage through 2 independent pathways - the trabecular meshwork and uveoscleral outflow pathway-determines the intra-ocular pressure. In patients with open-angle glaucoma, there is increased resistance to aqueous outflow through the trabecular meshwork. In contrast, the access to the drainage pathways is obstructed typically by their is in patients with angle-closure glaucoma ${ }^{[10]}$.

Intraocular pressure can cause mechanical stress and strain on the posterior structures of the eye, notably the lamina cribrosa and adjacent tissues. The sclera is perforated at the lamina where the optic nerve fibers (retinal ganglion cell axons) exit the eye. The lamina is the weakest point in the wall of the pressurized eye. Intraocular pressure-induced stress and strain may result in compression, deformation, and remodeling of the lamina cribrosa with consequent mechanical axonal damage and disruption of axonal transport that interrupts retrograde delivery of essential trophic factors to retinal ganglion cells from their brainstem target (relay neurons of the lateral geniculate nucleus) ${ }^{[11]}$.

Shi et al. ${ }^{[12]}$ reported a case of POAG in 25-years-old female patient. Ophthalmological examination supported diagnosis of POAG in both eyes. The case history and clinical data of the proband and her family members and followed standard genetic study procedures. In this family, there were 8 individuals ( 4 male and 4 female) diagnosed with POAG, 2 of whom died. Genetic analysis revealed that the inheritance pattern of POAG in this family is autosomal dominant.

\section{Conclusion}

Authors found that Primary open-angle glaucoma (POAG) is a disease that is often inherited. We reported case of familial POAG in 27 years old male patient who was managed successfully.

\section{References}

1. Quigley HA, Broman AT. The number of people with glaucoma worldwide in 2010 and 2020. $\mathrm{Br} \mathrm{J}$ Ophthalmol. 2006; 90:262-7.
2. Tham YC, Li X, Wong TY et al. Global prevalence of glaucoma and projections of glaucoma burden through 2040: a systematic review and meta-analysis. Ophthalmology. 2014; 121:2081-90.

3. Friedman DS, Wolfs RC, O'Colmain BJ et al. Prevalence of open-angle glaucoma among adults in the United States. Arch Ophthalmol. 2004; 122:532-8.

4. Rudnicka AR, Mt-Isa S, Owen CG et al. Variations in primary open-angle glaucoma prevalence by age, gender, and race: a Bayesian meta-analysis. Invest Ophthalmol Vis Sci. 2006; 47:4254-61.

5. Foster PJ, Buhrmann R, Quigley HA et al. The definition and classification of glaucoma in prevalence surveys. Br J Ophthalmol. 2002; 86:238-42.

6. Stroup DF, Berlin JA, Morton SC et al. Meta-analysis of observational studies in epidemiology: a proposal for reporting. Meta-analysis Of Observational Studies in Epidemiology (MOOSE) group. JAMA 2000; 283:2008-12.

7. Lunn D, Spiegelhalter D, Thomas A et al. The BUGS project: Evolution, critique, and future directions. Stat Med. 2009; 28:3049-67.

8. Friedman DS, Jampel HD, Munoz B et al. The prevalence of open-angle glaucoma among blacks and whites 73 years and older: the Salisbury Eye Evaluation Glaucoma Study Arch Ophthalmol. 2006; 124:1625-30.

9. Tielsch JM, Sommer A, Katz J et al. Racial variations in the prevalence of primary open-angle glaucoma. The Baltimore Eye Survey. JAMA 1991; 266:369-74.

10. Chaturvedi N. Ethnic differences in cardiovascular disease. Heart 2003; 89:681-6.

11. Klein R, Klein BE. The prevalence of age-related eye diseases and visual impairment in aging: current estimates. Invest Ophthalmol Vis Sci. 2013; 54:5-13.

12. Shi K, Zhao L, Wang CY. Familial primary open-angle glaucoma: a case report. Genetics and Molecular Research. 2014; 13(2):3162-4. 